\title{
Atypical Presentation of Celiac Disease: A Case Report
}

\author{
Kumar S. ${ }^{1}$, Sharma A. ${ }^{2}$ \\ ${ }^{1}$ Dr. Sunil Kumar, Senior Resident, Department of Pediatrics, Kalpana Chawla Government Medical College, Karnal, \\ Haryana, ${ }^{2}$ Dr. Amit Sharma, Consultant Gastroenterologist, Sanjeev Bansal Cyguns Hospital, Karnal, Haryana, India.
}

Corresponding Author: Dr. Sunil Kumar, Senior Resident, Department of Pediatrics, Kalpana Chawla Government Medical College, Karnal, Haryana, India. Email: drsunilx@yahoo.com

\begin{abstract}
Celiac disease is a chronic inflammatory immune-mediated condition associated with small intestinal injury triggered by gluten present in wheat, barley and rye, leading to malabsorption of different nutrients. Usually it presents as young age at the time of introduction of cereals with symptoms of malabsorption like diarrhea, abdominal distension and failure to thrive. But celiac disease has a broad range of general, gastrointestinal and systemic presentation. We hereby present a case of celiac disease presenting as a case of refractory vomiting, responding dramatically to gluten free diet.
\end{abstract}

Keywords: Celiac disease, Gastritis, Vomiting

\section{Introduction}

Celiac disease is an immune-mediated enteropathy triggered by the ingestion of gluten-containing grains in genetically susceptible individuals. $\mathrm{CD}$ is associated with HLA molecules DQ2 (90\%-95\%) and DQ8 (5\%$10 \%) \mathrm{CD}$ is one of the most common lifelong disorders worldwide and is characterized by a variety of clinical presentations. These include the typical malabsorption syndrome (classic symptoms) and a spectrum of symptoms potentially affecting any organ or body system (non-classic symptoms) [1].

\section{Case Report}

XYZ, 15 years old girl was brought to us with complaints of fever and vomiting associated with epigastric pain for one week, for which she was being treated at a private hospital with IV antibiotics, Ondasterone and proton pump inhibitors. Fever relived with the treatment but vomiting persisted.

Vomiting was non-bilious, non-projectile, not blood mixed and was not associated with significant systemic symptoms like headache or diarrhea.

She had no significant past history of any gastrointestinal symptoms or any chronic systemic disease and no significant family history of any gastrointestinal or systemic disease.

She did have atopic dermatitis with dermatographism. Her general examination was unremarkable except for reduced weight and height for age (4 and 23 percentile as per WHO CDC data) and mild pallor.

Mild epigastric tenderness was noted on abdominal examination. Fundus and blood pressure was normal with normal neurological examination.

Routine investigation showed moderate anemia with blood picture suggestive of iron deficiency (Hb $8.5 \mathrm{gm} / \mathrm{dl}$, MCV $83.1 \mathrm{fL}, \mathrm{MCH} 24.0 \mathrm{pg}$, RBC Count $3.5 \times 10^{6} / \mathrm{mm}^{3}$, RDW 17.1\%.).

Liver enzymes were found to be mildly elevated (AST: 65 IU/L, ALT: 71 IU/L). USG abdomen was also non remarkable.

Manuscript received: $28^{\text {th }}$ September 2018

Reviewed: $8^{\text {th }}$ October 2018

Author Corrected: $14^{\text {th }}$ October 2018

Accepted for Publication: $17^{\text {th }}$ October 2018 

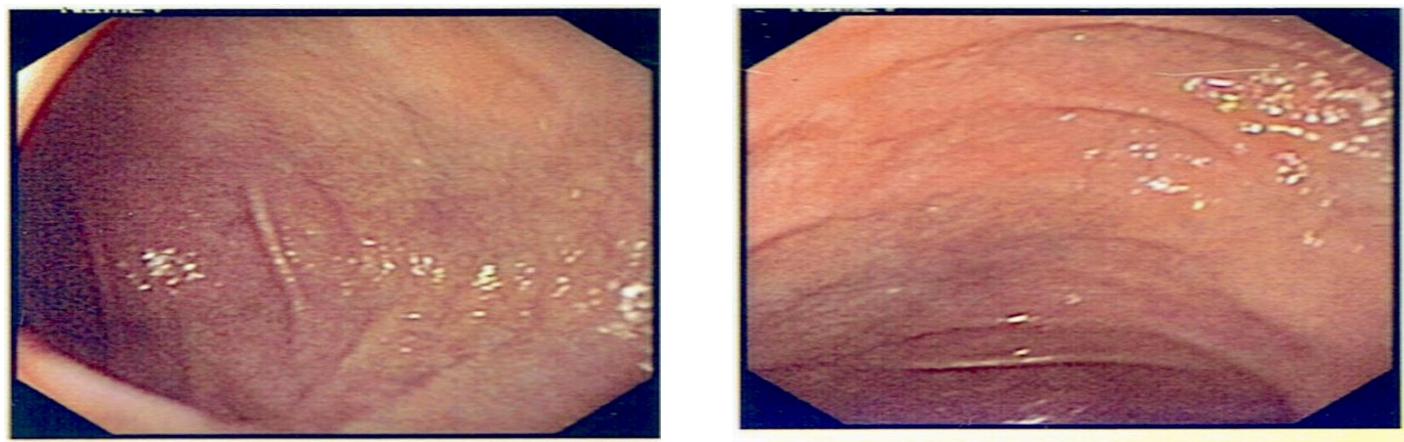

Figure-1: Endoscopy of patient showing loss of duodenal folds

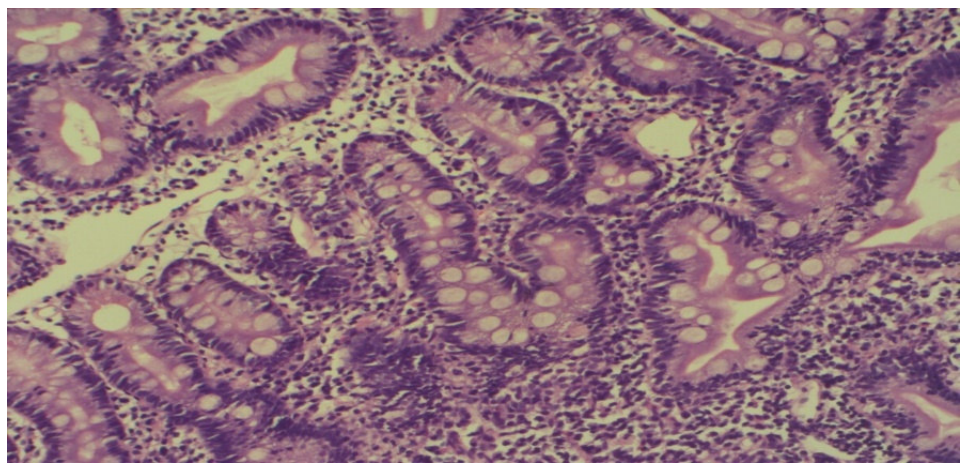

Figure-2: Histopathological picture of the duodenal biopsy showing changes suggestive of celiac disease viz. partial villous atrophy, hyperplasia of crypts, with increased intraepithelial lymphocytosis (Marsh IIIa)

Provisional diagnosis of gastritis Trial of different antiemetic (Ramosetron, Metoclopromide, Promethazine) was given with antacids with no significant improvement in vomiting.

UGI endoscopy was planned in view of refractory vomiting which showed markedly reduced height and number of duodenal folds. A biopsy was taken and histopathology examination was conducted. Histopathology was suggestive of celiac disease with partial villous atrophy, hyperplasia of crypts, with increased intraepithelial lymphocytosis (Marsh IIIa). Anti tTG was found to be positive.

Patient was put on gluten free diet and responded dramatically. Symptoms were reduced drastically and within 3 days IV medications were stopped and oral medication were stopped within 1 week. This improvement was maintained on follow-up visits.

\section{Discussion}

Celiac disease can have a wide spectrum of clinical presentation [2-7].

Classically celiac disease presents as gastrointestinal manifestations chronic diarrhea, abdominal distention, poor appetite leading to impaired growth, muscle wasting and hypotonia after the introduction of gluten in the diet in infancy i.e. 6 months- 2 years. Children can also present dramatically as celiac crisis, presenting with explosive watery diarrhea, marked abdominal distension, dehydration, electrolyte imbalance, hypotension, and lethargy. Despite a wide variability between countries, typical CD still represents a common presentation in the pediatric age group. Celiac disease may present withon-classic picture with delayedonset of symptoms involving older children (57 years old) with unusual intestinal complaints (e.g., recurrent abdominal pain, nausea, vomiting, bloating, and constipation) or extraintestinal manifestations (e.g., short stature, pubertal delay, iron deficiency, dental enamel defects).

Celiac disease can be clinically silent also, when typical gluten-sensitive enteropathy in absence of any clinical feature of celiac disease found on screening of persons of at-risk groups (diabetes and first-degree relatives) or 
general population in screening programs. However careful clinical investigation finds thatmany of these silent cases are indeed affected with alow-grade illness often associated with decreased psychophysical wellbeing.

A potential form of $\mathrm{CD}$ is diagnosed in patients who have anti-endomysium antibodies (AEA) and/orantihuman tissue transglutaminase antibodies, the typical HLA- predisposing genotype (DQ2 or DQ8), but anormal or minimally abnormal mucosal architecture (increased intraepithelial count) at the intestinal biopsy examination. These patients are at risk for developing atypical CD enteropathy later in life.

As celiac disease can present at any age have a variety of atypical clinical presentations like in this case, its diagnosis is likely to be missed. Undetected celiac disease can lead to long term complications like anemia, failure to thrive, osteoporosis, infertility, cancer and neurological complications like ataxia and depression [8]. These complications can be avoided by keeping a high index of suspicion in case presenting with nonspecific gastrointestinal symptom, not typical of celiac disease.

\section{Conclusion}

This reports highlights variety of symptoms through which celiac disease can present and need to have a high index of suspicion to avoid missing diagnosis and dreaded long term complications of this lifelong disease.

Funding: Nil, Conflict of interest: None initiated, Perission from IRB: Yes

\section{References}

1. Fasano A. Clinical presentation of celiac disease in the pediatric population. Gastroenterology. 2005 Apr; 128 (4 Suppl 1): S68-73.

2. Catassi C, Fabiani E, Rätsch IM, et al. The celiac iceberg in Italy. A multi-centre antigliadin antibodies screening for coeliac disease in school-age subjects. Acta Paediatr Suppl. 1996 May;412:29-35.

3. Hill I, Fasano A, Schwartz R, et al. The prevalence of celiac disease in at-risk groups of children in the United States. J Pediatr. 2000 Jan;136(1):86-90.

4. Hoffenberg EJ, Mac Kenzie T, Barriga KJ, et al. A prospective study of the incidence of childhood celiac disease. J Pediatr. 2003 Sep;143(3):308-14.

5. Fasano A, Berti I, Gerarduzzi T, et al. Prevalence of celiac disease in at-risk and not-at-risk groups in the United States: a large multicenter study. Arch Intern Med. 2003 Feb 10;163(3):286-92.

6. Mäki M, Mustalahti K, Kokkonen J, et al. Prevalence of Celiac disease among children in Finland. N Engl J Med. 2003 Jun 19;348(25):2517-24.

7. Catassi C, Fasano A. New developments in childhood celiac disease.Curr Gastroenterol Rep 2002; 4:238-243. DOI: https://doi.org/10.1007/s11894-0020069-0

8. Fasano A, Catassi C. Current approaches to diagnosis and treatment of celiac disease: an evolving spectrum. Gastroenterology. 2001 Feb;120(3):636-51.

\section{How to cite this article?}

Kumar S, Sharma A. Atypical Presentation of Celiac Disease: A Case Report. Int J Pediatr Res.2018;5(10):487-489. doi:10.17511/ijpr.2018.i10.02. 